\title{
Thermal Compensation Model of Magnetic Circuits with Modern Magnetic Materials
}

\author{
T. Szumiata*And M. GziK-Szumiata \\ Department of Physics, University of Technology and Humanities in Radom, \\ Krasickiego 54, 26-600 Radom, Poland
}

\begin{abstract}
In this work a quantitative analysis of thermal compensation has been performed for a magnetic circuit producing magnetic field in the air gap. The considered system consists of $\mathrm{Sm}_{3} \mathrm{Co}_{17}$ type permanent magnet (as a source of magnetic field), nanocrystalline FINEMET alloy (as ultra-soft magnetic medium) and Fe-Ni low Curie temperature compensative material (as a magnetic shunt). Distribution of magnetic field induction in the circuit has been calculated numerically within standard one-dimensional approximation, considering nonlinearities of compensative material as well as demagnetization susceptibility of permanent magnet. It has been theoretically predicted, that an appropriate choice of the compensative element thickness improves significantly thermal stability of magnetic field in the air gap.
\end{abstract}

DOI: $10.12693 /$ APhysPolA.127.650

PACS: $75.50 . \mathrm{Ww}, 75.50 . \mathrm{Vv}, 75.50 . \mathrm{Kj}, 75.50 . \mathrm{Bb}, 07.55 . \mathrm{Db}$

\section{Introduction}

Over the past two decades a great progress has been achieved in permanent magnets technology. Tradition Alnico@ and ferrite-type magnets have been replaced by neodymium-containing and samarium-cobalt materials of much higher coercivity and resulting $(B H)_{\max }$ energy product. However these types of new magnets reveals relatively high values of reversal temperature coefficients (RTC) of remanent magnetization (or residual induction), which is not acceptable in metrology and other sophisticated applications such as gyroscopes, sensors, actuators and elementary particles colliders. Although there are numerous experimental papers devoted to the thermal compensation procedure of magnets in such systems (e.g. [1-7]), much less theoretical attempts for analysing this problem have been made till yet. A thermal compensation of magnetic circuits by use of dedicated materials is a basic "technical" way of the reduction of effective RTC of magnet remanence. On the other hand a physical analysis of remanence dependence on temperature would require not only a consideration of the evolution of spin-spin interactions but also a domain structure changes, which have not been reported in literature in satisfactory degree. The main goal of present work is to present relatively simple and universal model of thermal compensation of permanent magnets, which would be helpful in designing of magnetic circuits.

\section{Theoretical model}

The geometry of considered magnetic circuit is presented in Fig. 1. The system consists of the following elements: permanent magnet (A), soft magnetic material as magnetic flux guide (B), a plate of low Curie temperature material as a compensating magnetic shunt $(\mathrm{C})$

\footnotetext{
${ }^{*}$ corresponding author; e-mail: t.szumiata@uthrad.pl
}

and air gap (D). Such planar circuit could be a part of e.g. electric energy meters, tachometer or calibration systems for hallotron sensors. The operational aim of this work is to find an optimal thickness $d$ and position $x$ of compensating plate in order to achieve the best thermal stability of the magnetic induction $\mathrm{B}_{6}$ in the air gap of given width $g$.

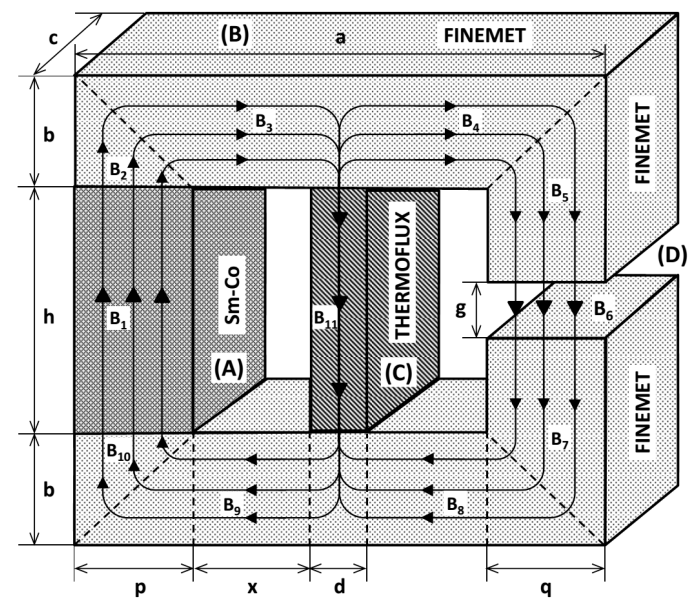

Fig. 1. Scheme of thermally compensated magnetic circuit.

From Gauss law for the magnetic flux one obtains followings relations between magnetic induction values in different parts of magnetic circuit after consideration of its symmetry:

$$
\begin{aligned}
& B_{2} p-B_{3} b=B_{3} b-B_{4} b-B_{11} d=B_{4} b-B_{5} q=0, \\
& B_{1}=B_{2}, \quad B_{5}=B_{6}=B_{7}, \quad B_{4}=B_{8}, \\
& B_{3}=B_{9}, \quad B_{2}=B_{10}
\end{aligned}
$$

Ampère laws for main contour of the magnetic circuit and the contour containing the compensating shunt give following two relations, respectively: 


$$
\begin{aligned}
& H_{1} h+H_{2} \frac{b}{2}+H_{3}\left(\frac{p}{2}+x+\frac{d}{2}\right)+H_{6} g+H_{10} \frac{b}{2} \\
& +H_{4}\left(a-p-\frac{q}{2}-x-\frac{d}{2}\right)+H_{5}\left(\frac{h-g}{2}+\frac{b}{2}\right) \\
& +H_{7}\left(\frac{h-g}{2}+\frac{b}{2}\right)+H_{8}\left(a-p-\frac{q}{2}-x-\frac{d}{2}\right) \\
& +H_{9}\left(\frac{p}{2}+x+\frac{d}{2}\right)=0, \\
& H_{1} h+H_{2} \frac{b}{2}+H_{3}\left(\frac{p}{2}+x+\frac{d}{2}\right)+H_{10} \frac{b}{2}+H_{11} h \\
& +H_{9}\left(\frac{p}{2}+x+\frac{d}{2}\right)=0,
\end{aligned}
$$

where $H_{i}$ are the values of magnetic field strength in the individual regions of magnetic circuit.

Material relation in the inverse form, i.e. $H(B)$ more convenient for further calculations - is assumed to be nonlinear. In the case of $(\mathrm{B})$ and $(\mathrm{C})$ material it is represented for the sake of simplicity as composition of three linear functions:

$$
H(B)=\left\{\begin{array}{l}
\frac{B}{\mu_{r}} \quad \text { for } B<\mu_{\mathrm{r}} \mu_{0} H_{\mathrm{m}} \\
\frac{B-\left(\mu_{\mathrm{r}}-\mu_{\mathrm{m}}\right) \mu_{0} H_{\mathrm{m}}}{\mu_{\mathrm{m}}} \\
\begin{array}{l}
\text { for } \mu_{\mathrm{r}} \mu_{0} H_{\mathrm{m}} \leq B \leq\left(\mu_{\mathrm{r}}-\mu_{\mathrm{m}}\right) \mu_{0} H_{\mathrm{m}} \\
+\mu_{\mathrm{m}} \mu_{0} H_{\mathrm{s}}
\end{array} \\
\frac{B-\left(\mu_{\mathrm{r}}-\mu_{\mathrm{m}}\right) \mu_{0} H_{\mathrm{m}}-\left(\mu_{\mathrm{m}}-\mu_{\infty}\right) \mu_{0} H_{\mathrm{s}}}{\mu_{\infty}} \\
\text { for } B>\left(\mu_{\mathrm{r}}-\mu_{\mathrm{m}}\right) \mu_{0} H_{\mathrm{m}}+\mu_{\mathrm{m}} \mu_{0} H_{\mathrm{s}}
\end{array}\right.
$$

where $\mu_{\mathrm{r}}, \mu_{\mathrm{m}}, \mu_{\infty}$ are relative magnetic permeabilities in low field region $\left(H \leq H_{\mathrm{m}}\right)$, medium field region $\left(H_{\mathrm{m}} \leq H \leq H_{\mathrm{s}}\right)$ and saturation region $\left(H \geq H_{\mathrm{s}}\right)$, respectively. For permanent magnet $(\mathrm{A})$ the $H_{1}\left(B_{1}\right)$ relation is set via magnetic susceptibility $\chi$ describing demagnetization influence on remanent magnetization $M_{\mathrm{r}}$ :

$$
M_{1}\left(B_{1}\right)=\frac{M_{\mathrm{r}}+\chi \frac{B_{1}}{\mu_{0}}}{1+\chi}, H_{1}\left(B_{1}\right)=\frac{B_{1}}{\mu_{0}}-M_{1} .
$$

Realistic values of material parameters have been incorporated into the model. VACOMAX $240 \mathrm{HR}$ - a new generation $\mathrm{Sm}_{3} \mathrm{Co}_{17}$-type permanent magnet has been considered. Information about magnetic parameters of this material is accessible on the website of the manufacturer (Vacuumschmelze [8]). For such magnet a reversible temperature coefficient of high remanent flux density $(1.12 \mathrm{~T})$ is relatively low in the temperature range close to room temperature $\left(\alpha=-3.0 \times 10^{-4} 1 /{ }^{\circ} \mathrm{C}\right)$ when compared with this of neodymium magnets, however for advanced applications the magnet still demands thermal compensation. As compensating material a THERMOFLUX 65/100-G Fe-Ni alloy with approx. 30\% nickel has been applied - well known for many years, but recently manufactured very precisely in the form of thin metal sheets by Vacuumschmelze [8]. This material is characterized by low Curie temperature $\left(T_{\mathrm{c}} \approx 65^{\circ} \mathrm{C}\right)$ and very high negative temperature coefficient of relative magnetic permeability $\left(\approx-0.031 /{ }^{\circ} \mathrm{C}\right)$. As a "transmitter" of magnetic flux a magnetically ultra-soft nanocrystalline FINEMET-type alloy has been considered. It was invented by Yoshizawa, Oguma and Yamauchi [9] in late eighties and now commercially manufactured by HITACHI Metals Ltd [10]. Structure and magnetic phenomena in such material was carefully studied by Herzer [11, 12] from Vacuumschmelze [8] and manufactured as VITROPERM. The role of interface region of grains in nanocrystalline FINEMET-like materials was examined by Szumiata et al. [13] with Mössbauer spectrometry and strain modulated ferromagnetic resonance (SMFMR) method of magnetostriction measurements. The most important features of FINEMET for present purpose are: its very high saturation flux density $(\approx 1.2 \mathrm{~T})$ in low magnetic field, large initial magnetic permeability $\left(\approx 2.5 \times 10^{5}\right)$ revealing low, positive value of thermal coefficient $\left(\approx 7 \times 10^{-4} 1 /{ }^{\circ} \mathrm{C}\right)$ in vicinity of room temperature.

A system of equations (1) and (2) including nonlinear material relation (3) and linear correction for permanent magnet (4) has been solved numerically with residuum minimizing method by applying both gradient and evolutionary algorithms. It is relatively simply to implement using software like PTC Mathcad or even MS Excel with Solver component. The following dimensions of individual elements of magnetic circuit have been assumed in calculations: $h=5 \mathrm{~cm}, a=10 \mathrm{~cm}, b=p=2 \mathrm{~cm}$ (a value of thickness $c$ is not important in the case of planar symmetry). The most expected results are $B_{6}$ values of magnetic flux density in air gap for several temperatures, which enable to estimate a RTC value of $B_{6}$. Within a numerical procedure it has been calculated as a change of $B_{6}$ related to its value at room temperature and divided by the change of the temperature.

\section{Results}

In Fig. 2a RTC values of $B_{6}$ magnetic induction in air gap are presented as a function of $d$ thickness of compensating magnetic shunt for several values of air gap width $g$. Without compensation $(d=0)$ RTC values are close to the value for permanent magnet itself. The form of $\mathrm{RTC}(d)$ dependencies seems to be almost linear. When $d \approx 0.3 \mathrm{~mm}$ all lines (for different widths of air gap) intersect in one point, where RTC takes noticeably smaller negative value than initial ones. However, for each value of $g$ width there is an individual, optimal value of the compensating shunt thickness $d_{\mathrm{opt}}$, which provides - at least theoretically - a full thermal compensation, i.e. zero value of RTC for magnetic flux density in air gap. The slope of RTC $(d)$ lines allows to predict that by controlling the thickness $d$ of compensating magnetic sheet with precision better than $0.1 \mathrm{~mm}$ it is possible to reduce RTC value more than 10 times in comparison to the $\alpha$ value of permanent magnet, i.e. the thermal magnetic compensation of $B_{6}$ will be of the order of $10^{-5} 1 /{ }^{\circ} \mathrm{C}$. As 
shown in Fig. 2b the side-effect of the application of compensating magnetic shunt is a reduction of magnetic flux density in the air gap, however the relative loss $\delta$ is only about $1 \%-3 \%$ for $d=d_{\text {opt }}$.
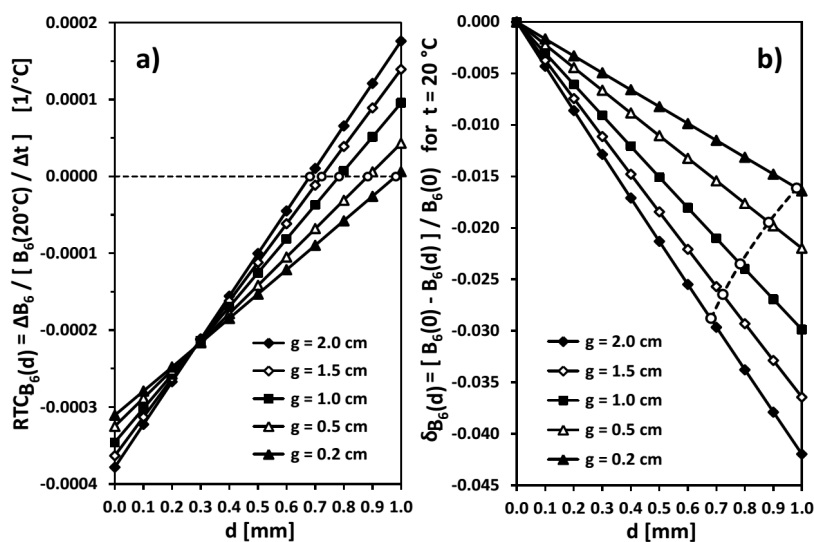

Fig. 2. a) Reversal temperature coefficient of magnetic field induction $B_{6}$ in air gap of compensated magnetic circuit and b) relative loss of magnetic field after compensation as a function of compensating plate thickness for different values of air gap width. Open circles correspond to the optimal thickness of compensating magnetic shunt. All lines are guides for the eye.
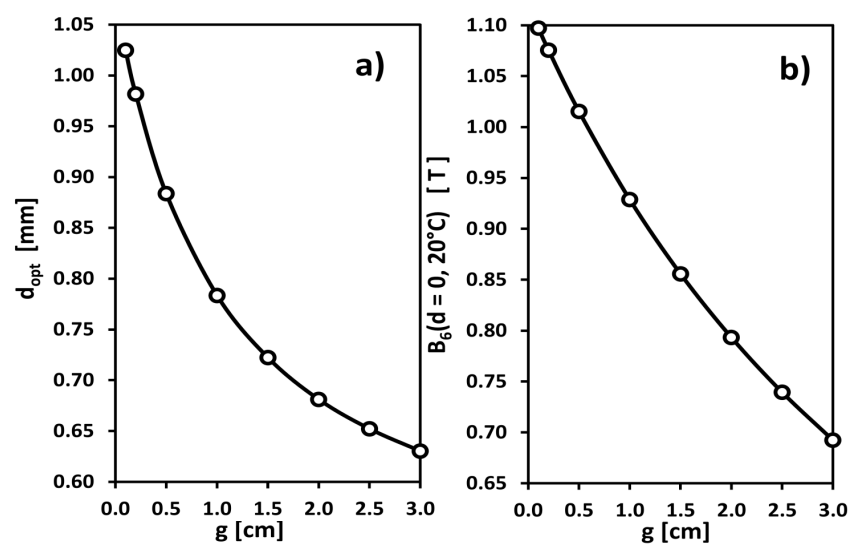

Fig. 3. a) Optimal thickness of compensating magnetic shunt and b) induction of magnetic film in air gap of non-compensated magnetic circuit as a function of air gap width (solid lines are guides for the eye).

Figure 3a illustrates the dependence of $d_{\text {opt }}$ on the air gap width. The narrower air gap one adjusts, the more difficult compensation is - i.e. it demands thicker compensating shunt, what results in greater relative loss of magnetic induction (according to Fig. 2b). However, as seen in Fig. 3b, the absolute values of magnetic flux density in the case of narrow gaps is significantly higher, thus - in practice - the loss caused by compensation will not be so crucial. It has been tested numerically, that all results described above are almost independent on the position $x$ of magnetic shunt.

\section{Conclusions}

Presented theoretical predictions indicate that temperature compensation procedure with appropriate magnetic shunt can significantly elevate a thermal stability of magnetic field in the air gap. The improvement can reach a factor of 10 (in comparison to thermal coefficient of permanent magnet remanence), when controlling precisely a thickness of the compensating metal sheet. Better thermal stability of magnetic flux density in the compensated circuit has been achieved at the expense of the loss of magnetic induction, but not greater than several percent. The model presented above can be helpful in a practical designing of thermally high-stable magnetic circuits. Its simplicity enables quick modifications and relatively fast calculations. Application of the sophisticated finite element method (FEM) would be a way of considering the nuances of 3D spatial distribution of magnetic flux density in the circuit. Nevertheless, dedicated computer programs for FEM simulations of magnetic systems usually need some adaptation for the problem of thermal magnetic compensation.

\section{References}

[1] K. Turek, J. Chmist, H. Figiel, J. Magn. Magn. Mater. 157-158, 65 (1996).

[2] S.H. Kim, C. Doose, Particle Accelerator Conference 3, 3227 (1997).

[3] K.R. Rajagopal, B. Singh, B.P. Singh, N. Vedachalam, IEEE Transactions on Magnetics 37, 1995 (2001).

[4] T. Mihara, Y. Iwashita, M. Kumada, A. Evgeny, Ch.M. Spencer, $S L A C-P U B-10248,1$ (2004).

[5] T. Mihara, Y. Iwashita, M. Kumada, E. Antokhin, E. Sugiyama, C.M. Spencer, SLAC-PUB-10876, 1 (2004).

[6] K. Bertsche, G.W.Foster, J-F. Ostiguy, IEEE Proc. 95CH35843, 1381 (1996).

[7] G.W. Foster, FERMILAB-CONF-98-423-AD Conf. Proc. C980622, 189 (1998).

[8] Vacuumschmelze.

[9] Y. Yoshizawa, S. Oguma and K. Yamauchi, J. Appl. Phys. 64, 6044 (1988).

[10] Hitachi Metals Ltd.

[11] G. Herzer, IEEE Trans. Magn. 26, 1397 (1990).

[12] G. Herzer, Hanbook of Magnetic Materials, Vol. 10, Chap. 3, Ed. K.H.J. Buschow, Elsevier Science, Amsterdam 1997, p. 415.

[13] T. Szumiata, K. Brzózka, M. Gawroński, B. Górka, J.S. Blázquez-Gámez, T. Kulik, R. Żuberek, A. Ślawska-Waniewska, J. Magn. Magn. Mater. 272-276, 1443 (2004). 